\title{
Suspension Type Stereo Bicycle Parking Garage
}

\author{
Zhang Jianmin, Zhou Jun, Liu Lei, Tian Peipei, Zhou Shijun \\ College of Mechanical Engineering, Shanghai University of Engineering Science, Shanghai, China
}

\section{Email address:}

jianminlvld@163.com (Zhang Jianmin)

\section{To cite this article:}

Zhang Jianmin, Zhou Jun, Liu Lei, Tian Peipei, Zhou Shijun. Suspension Type Stereo Bicycle Parking Garage. American Journal of Applied Scientific Research. Vol. 2, No. 6, 2016, pp. 55-58. doi: 10.11648/j.ajasr.20160206.14

Received: October 18, 2016; Accepted: November 5, 2016; Published: December 5, 2016

\begin{abstract}
At present, many countries have vigorously advocated the use of green environmental protection of the bicycle as a means of transport, a large number of bicycle parking problems become a problem. In order to solve the present bicycle parking space, parking chaos, and convenient use, easy theft problem, this paper proposes a suspension type stereo bicycle parking garage, mainly includes three parts: rotating device, lifting device, fingerprint lock device, through the combination of the three can achieve the design goal of each layer parking a number of parking spaces, and can realize every layer of parking spaces and parking spaces for each rise and fall, the design of fingerprint lock device to ensure the safety of the bicycle parking garage, and parking garage can achieve overall contraction, simple and portable.
\end{abstract}

Keywords: Suspension Type Stereo Bicycle Parking Garage, Rotation Device, Lifting Device, Fingerprint Lock Device

\section{Introduction}

With the progress of society and the improvement of people's living standard, into the rich era of cars, but because of the environmental problems and the city travel car have a car, let the people cognitive deficiency, and ushered in the green travel convenient bicycle tide, countries to advocate to use the bicycle as transportation. However, increasing the usage of the bicycle is to bring about a series of problems in the city park, such as parking space is small, easy to damage the appearance of the bicycle; [1-3] bicycle with Irregular arrangement, affect the appearance of the city; overlap, blocking traffic on the sidewalk and bicycle lane design; parking lax supervision, theft of high frequency, take the car to solve more trouble, also easy to accidentally knocked over the bike next door will fall into the problems caused by. [4-6] Bicycle parking problems become more and more concerned by people, has become an urgent problem to be solved.

In view of the above problems and the characteristics of different occasions, people have developed a bicycle parking device, such as ensuring the stability and safety of the bicycle and making full use of space and other functions. [7] At present, in schools, communities and other general will have a dedicated bike garage, but will not only take up a larger space, but also can not solve the existing problems of bicycle parking and access is not convenient. [8] Now the market also has a double layer of bicycle parking, but only to achieve a small number of bicycle placement problem, can not solve the problem of a large number of bicycle placement. In order to solve the problem of bicycle parking is facing, this paper designs a suspension type stereo bicycle parking, vehicles can not only many kinds of bicycle parking and access to the realization of simple and convenient, also designed a fingerprint lock device, to provide security for bicycle parking.

\section{The Overall Design of the Suspension Type Stereoscopic Bicycle Parking Garage}

\subsection{Overall Design}

This project is the design of a suspension type parking garage, which mainly includes the base, the rotating device, the lifting device and the fingerprint lock device. The parking garage for the three-dimensional hanging type bicycle parking garage, the design has two layers of parking spaces, can be extended to the multi-layer, each layer has a number of parking spaces, you can achieve the needs of many kinds of bicycle. The rotating device is fixed on the base, and the rise and fall of each layer of parking spaces is realized through the coordination of the rotating device and the lifting device. The 
lifting device is arranged with a plurality of parking spaces, which can realize the rise and fall of the designated parking spaces. [9-10] Fingerprint lock device through the PLC programming to achieve each parking bike lock and unlock.

Drawings are as follows: 1:Base, 2:Rotating device, 3:Lifting device, 4:Electric machinery, 5:Wire rod, 6:Coupling, 7:Floor, 8:Rotary sleeve, 9:Transverse bracing, 10:Linear actuator, 11:Round table, 12:Groove, 13:Card slot, 14:Through hole, 15:Circlip, 16:Hook, 17:Rings, 18:Earrings, 19:Cross bar, 20:Hook A, 21:Hook B, 23:Fingerprint lock, 24:Fingerprint identification module, 25:Operation panel, 26:Electronic lock, 27:Limit rod.

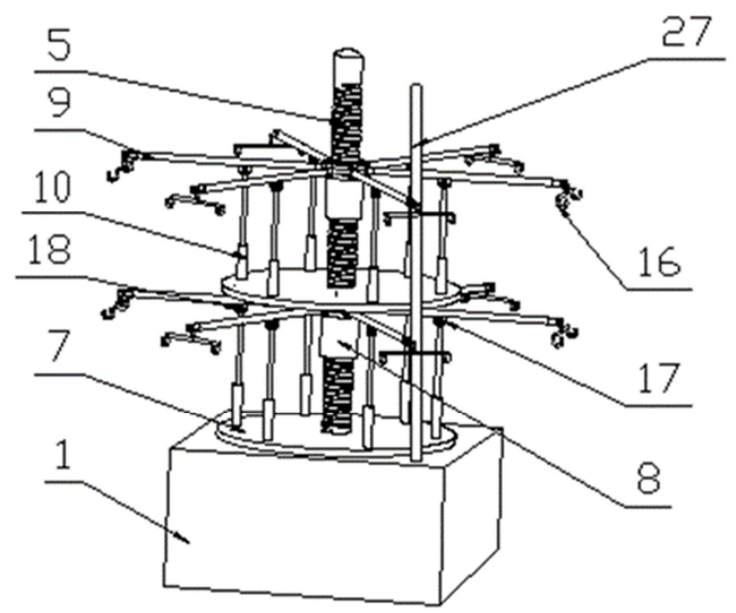

Fig. 1. The overall structure of the suspended solid bicycle parking garage.

\subsection{Design of Each Part}

1) Rotating device

The rotation device is designed, and the rise and fall of each layer of parking spaces are realized through the cooperation of the rotating device and the lifting device.

The rotating device mainly comprises a motor, a bottom plate and a screw rod. The motor is arranged in the base, and connected by a wire coupling rod; the bottom plate is round plate, placed in the center of the base; the screw rod is vertically arranged on the base, the middle position of the base is provided with threaded holes corresponding to the screw rod, the base through a threaded hole is sheathed on the screw rod. The base is provided with a finite position bar.

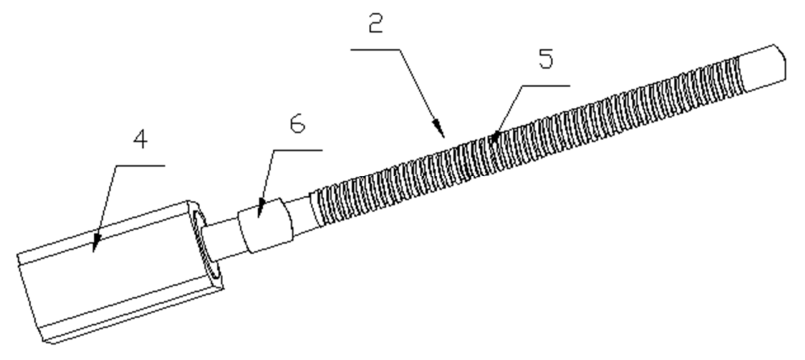

Fig. 2. Schematic diagram of rotating device structure.

2) Lifting device

Design of lifting device, lifting device to the last week of each layer provided with a plurality of parking spaces, the lifting device and the rotating device to cooperate with each other to achieve each layer of parking spaces in the rise and fall, the design of electric push rod lifting device can be achieved on the rise and fall of each parking space.

The lifting device mainly comprises a circular bottom plate, a rotary sleeve arranged on the screw rod and a transverse support arranged on the rotary sleeve. The middle position of the base plate is provided with threaded holes corresponding to the wire rod, plate through the screw hole is sheathed on the screw rod, the bottom plate is arranged on the lower part of the rotary sleeve; the rotary sleeve is a hollow cylinder, the inner side is provided with a screw thread sleeve, a rotary sleeve is provided with a cone, cone for air cylinder type, there are grooves are evenly arranged on the end surface of cone, cone outer side is provided with a clamping groove, rotating cone inner diameter is equal to the inner diameter of the sleeve; both ends of transverse brace is provided with a through hole groove is arranged in the clamping ring, clamping ring passes through the through hole and the cross brace are arranged in the groove hole, the other end of the transverse brace the hook is arranged. Cross brace is arranged on the lateral wall of the rings, the push rod electric push rod is arranged on the earrings, earrings and rings are arranged in the bolt. Start the electric push rod corresponding to the specified bicycle, electric push rod drives rod up and down, to achieve the specified bicycle up and down.

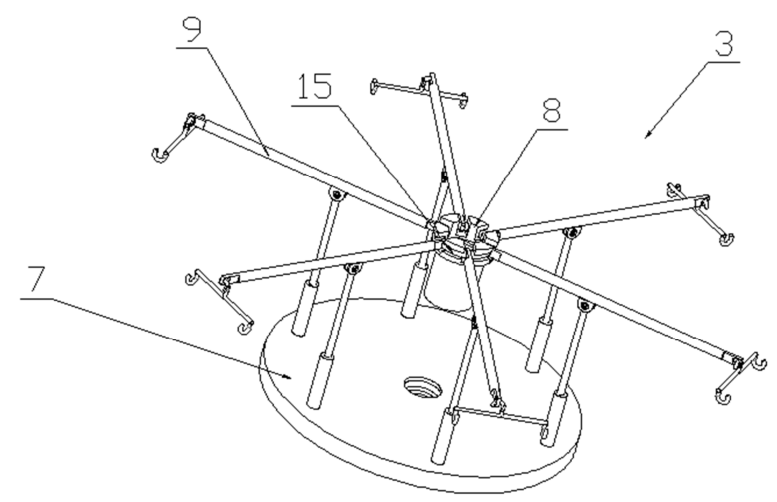

Fig. 3. Schematic diagram of rotating device structure.

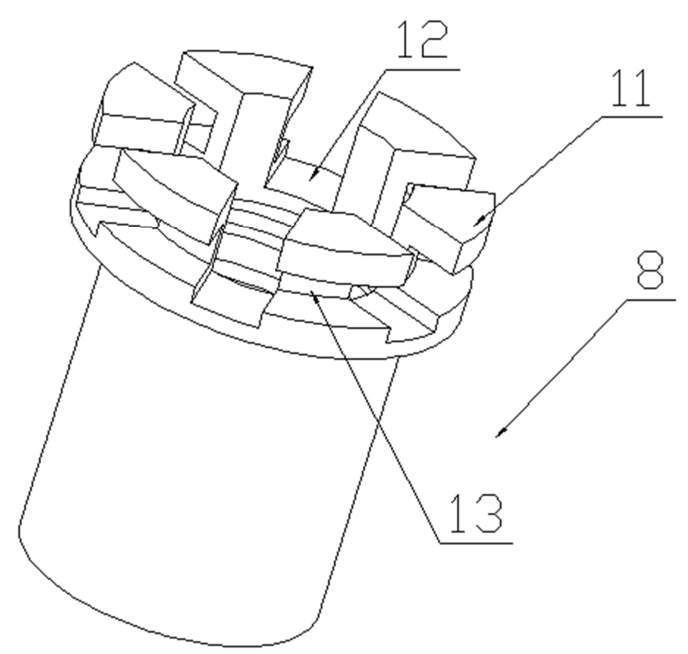

Fig. 4. Schematic diagram of the rotating sleeve. 


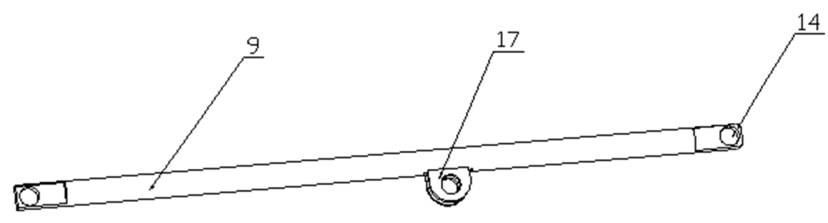

Fig. 5. The structure of the transverse brace.

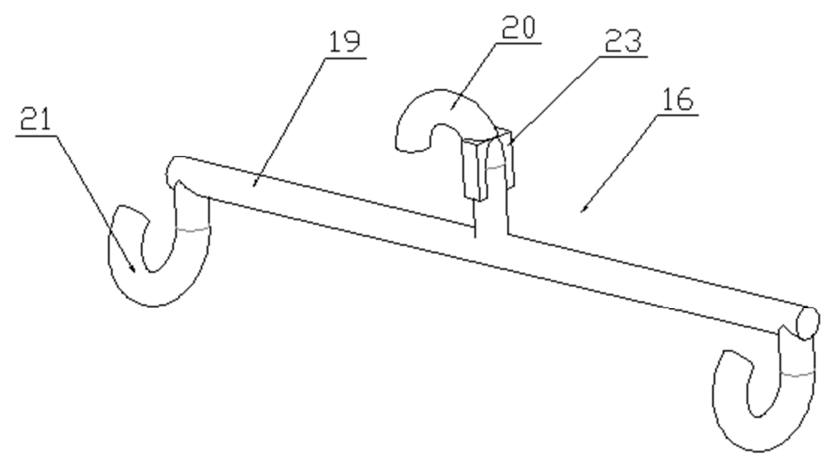

Fig. 6. Schematic diagram of the structure of the hook.

3) Fingerprint lock device

Fingerprint lock device design, through the PLC programming on each of the parking spaces on the fingerprint lock and unlock the bike. The fingerprint lock device is arranged on the hook of the lifting device, which comprises a fingerprint identification module, an operation panel and an electronic lock three parts. After parking the bicycle, the fingerprint lock is opened, and the parking space is locked to ensure the safety of the bicycle. When taking a bicycle, use the fingerprint to unlock and take off the bicycle.

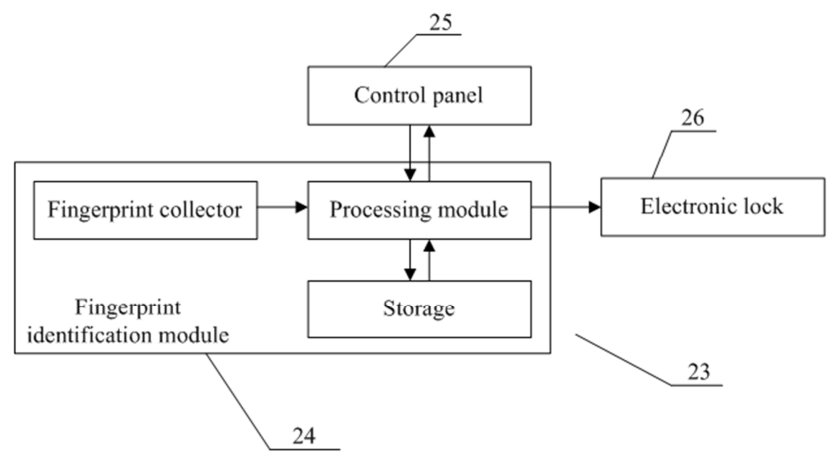

Fig. 7. Working principle of fingerprint lock device.

\section{Working Process}

When the bicycle in the off parking lot, first pick the first layer on the bicycle, start the motor, the motor drives the screw rod to reverse, at this time, the rotary sleeve with the screw rod to rotate, when the rotary sleeve of the supporting rod on the rotating limiting rod, the rotary sleeve and the wire rod can not rotate at the same time, at this time, the rotary sleeve on the screw rod to move downwards, at the same time, lifting device of other layers also toward the base direction, specify the desired bicycle, electric putter to start the corresponding bicycle, electric push rod drives pole descend slowly, until the bike completely open landing, fingerprint lock, bicycle will be removed, when the first the bike completely removed after the first layer of the electric push rod fully contracted, the first layer of the lifting device contraction lifting device second layer decrease with the first layer of the wire rod landing To a certain height, specify the desired bike after the start of the corresponding electric putter bicycle, electric putter drive rod falling slowly, until the bike completely start landing, electronic lock, bicycle will take when parking on the bicycle; completely removed after the parking contraction.

When the bicycle hanging in the parking lot, hang second bicycles, bicycle will be hung on the hook, close the fingerprint lock, electric push rod of the bicycle corresponding rises slowly, so the electric push rod rises to the maximum stroke, close the electric putter, and two layer is completely covered with a bicycle, bicycle are hung in the first layer, and then open the motor, the motor is transferred to drive the screw forward, when the rotary sleeve of the supporting rod on the rotating limiting rod, the rotary sleeve and the wire rod can not rotate at the same time, at this time, the rotary sleeve on the screw rod moves upward, each layer of the rotary sleeve moves upward until a certain height.

\section{Advanced Technology}

The project design of the hanging type solid bicycle garage can conveniently realize the bicycle parking and access, solves the problem that the existing bicycle parking and taking the messy and inconvenient problem, reached the advanced level, and the use of simple and convenient, stable working performance.

The technological advance of the design is mainly manifested in the following four aspects:

1) the suspension type stereo bicycle parking garage through the rotation device and the lifting device of the cooperation, the realization of the suspension of the bicycle parking and access, space saving and easy operation;

2) The suspension type three-dimensional bicycle parking garage design and design of the upper and lower two layers, can be extended to the multi-layer, can achieve more bicycle parking;

3) The hanging type stereo bicycle parking garage can realize the fingerprint locking and unlocking of the designated bicycle by the design of the fingerprint lock device, which increases the safety of the bicycle parking;

4) The hanging type solid bicycle parking garage can be through the lifting device and landing electric putter retraction, realize the contraction of the hanging type solid bicycle garage of the whole, which is convenient to carry and place.

\section{Summary and Outlook}

This paper gives a detailed description of the structure of hanging type solid bicycle garage, working process of the parking garage to summarize, the design solves the current mess, bicycle parking space and parking and convenient use, 
easy to be stolen, matched by a rotating device, lifting device, fingerprint lock device can be achieved parking design goal of each layer a number of parking spaces, and can realize every layer of parking spaces and parking spaces for each rise and fall, the design of fingerprint lock device to ensure the safety of the bicycle parking garage, and parking garage can achieve overall contraction, simple and portable. As a kind of bicycle parking garage, the hanging type solid bicycle garage is a kind of equipment with good development prospect, which is intelligent, automated, three-dimensional, bicycle parking system and a large amount of storage, access and other fast, anti-theft functions is the future city has a very competitive and development potential of a bicycle parking device, and is likely to be the mainstream in the future city bicycle parking device.

\section{References}

[1] Wang Qiuping, Xue Jian, Pei Xiaoli. Study on the layout of the connection facilities of bicycle and subway in Xi'an city [J]. traffic standardization, 2009 (11): 210-213.

[2] Wang Zhigao, Liu Daizong. Planning and design elements of bicycle parking facilities [J]. urban traffic, 2014,12 (4): 27-36.

[3] Liu handsome. Study on the planning and management model of bicycle parking facilities in Wuhan city [J]. traffic and transportation (Academic Edition), 2011 (7): 102-106.

[4] He Baohong, Li Yongchun, Li Xuefeng. The international experience of the development of bicycle traffic and the research status of China [J]. international urban planning, 2015, 30 (5): 104-109.

[5] Ge Cuiyu, bear dongwook. Analysis of the current situation of campus bicycle parking and Improvement Countermeasures a case study of Nanjing Institute of Technology [J]. Journal of Nanjing Institute of Technology (NATURAL SCIENCE EDITION), 2011, 9 (1): 68-72.

[6] Liu Lijun, Sultan. Tianjin city residential structure from the perspective of research on bicycle trip characteristics in Huayuan residential area of Tianjin urban construction college. Based on the cases of [J]. 2016, 22 (4): 248-253.

[7] Liu Du. Design of underground bicycle garage in Middle School of Shanxi architecture, 2012, 38 (29):8-11.

[8] Deng. Double deck bicycle parking system: China.10107034, 2 [P]. 2005-5-25.

[9] Li Peng, Liu Shuang. PLC theory and application technology [M]. Beijing: Publishing House of electronics industry, 2015.

[10] Zhang Bingqian, Liu Aiying, Feng Junping. The return to the green travel of bicycles and Countermeasures [J]. cooperative economy and science and technology, 2016 (10): 9-11. 